\title{
Combined irradiation and low-dose cyclophosphamide in the treatment of Graves' ophthalmopathy
}

\author{
Robert Teoh and Jean Woo
}

Department of Medicine, Chinese University of Hong Kong, Prince of Wales Hospital, Shatin, NT, Hong Kong.

\begin{abstract}
Summary: The treatment of a patient with euthyroid Graves' ophthalmopathy is described. A poor response was obtained from large doses of prednisone whereas a combination of orbital irradiation and low dose cyclophosphamide induced a dramatic resolution. As there is no fully satisfactory treatment for this condition, this combined therapy deserves further investigation.
\end{abstract}

\section{Introduction}

Ophthalmic Graves' disease may develop in the abscence of clinical or biochemical evidence of thyrotoxicosis and because of its immunological basis, autoimmune ophthalmopathy has been suggested as a better name. ${ }^{1}$ As the disease may represent a distinct autoimmune disorder, ${ }^{2}$ different forms of treatment aimed at immunomodulation have been used, such as steroids, azathioprine, cyclophosphamide, irradiation, plasmapheresis, and cyclosporin. Response to these treatments is variable and none is uniformly successful. ${ }^{1,3-7}$

We describe a patient with steroid-resistant autoimmune ophthalmopathy which responded dramatically to cyclophosphamide and a two week course of orbital irradiation. As far as we are aware, the successful use of this combination has not been previously reported.

\section{Case report}

A 26 year old female was referred in September 1983 with bilateral painful proptosis, chemosis and double vision. Three and a half months before admission pain, swelling and redness of the right eye developed. This was followed a month later by diplopia and proptosis of both eyes.

On examination, she had mild proptosis and severe chemosis. The visual axes were parallel but there was asymmetrical restriction of eye movements in all directions of gaze, the most affected being right lateral gaze. Visual acuity, fields, blind spot and optic fundi were all normal. Computed tomographic (CT) scan showed gross swelling of the extra-ocular muscles

Correspondence: Jean Woo.

Accepted: 30 July 1986 particularly of the left medial and lateral recti (Figure 1).

Routine blood tests were normal. Serum thyroxine $\left(\mathrm{T}_{4}\right)$ was $80 \mathrm{nmol} / 1$ (normal range $58-154 \mathrm{nmol} / \mathrm{l}$ ), Triiodothyronine $\left(\mathrm{T}_{3}\right.$ ) uptake $29.9 \%$ (range $25-35 \%$ ). Thyroglobulin and thyroid microsomal antibody titres were less than 1:10 and 1:100 respectively. The baseline thyroid stimulating hormone (TSH) was $1.0 \mathrm{mU} / 1$ and showed a normal rise to $4.4 \mathrm{mU} / 1$ thirty minutes after the i.v. injection of thyrotrophin releasing hormone (TRH) $(0.2 \mathrm{mg})$.

Prednisone $40 \mathrm{mg} /$ day was started. Initially the

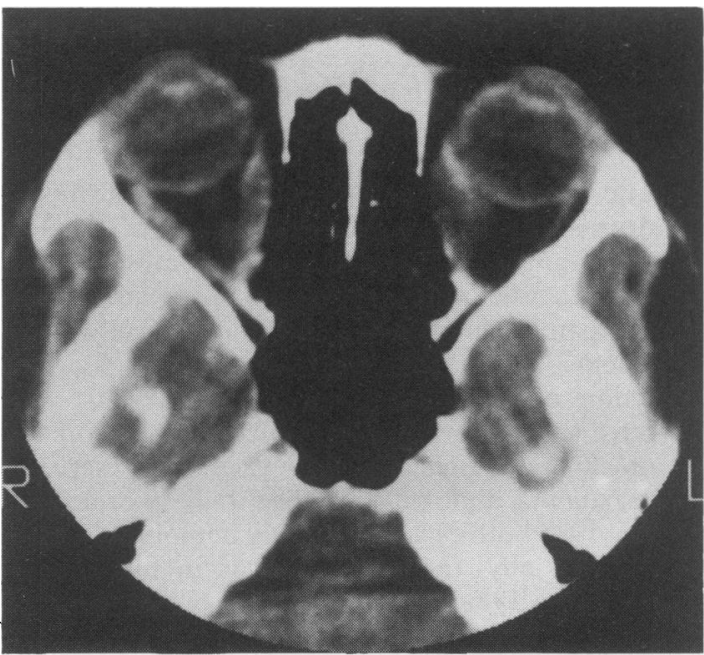

Figure 1 CT scan of the orbit before irradiation, showing swelling of the extraocular muscles.

(C) The Fellowship of Postgraduate Medicine, 1987 

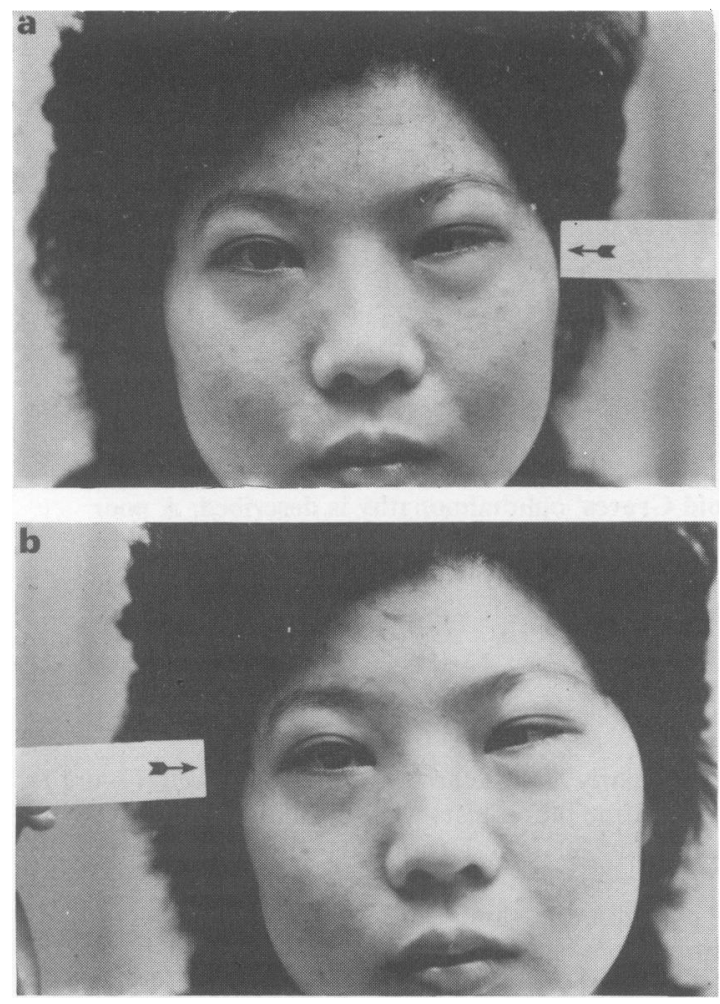

Figure 2 Eye movements before irradiation: (a) patient looking to her right; (b) patient looking to her left.

pain, proptosis and chemosis subsided though the diplopia persisted. As severe Cushingoid features developed with marked acne, hypertension and weight gain, the dose of prednisone was gradually reduced to $10 \mathrm{mg}$ over the next two to three months. However, on this dose, the diplopia, proptosis and chemosis deteriorated, the prednisone was increased to $40 \mathrm{mg} /$ day and frusemide, L-thyroxine and hydrocortisone eye drops were added. A repeat CT scan revealed further enlargement of the extraocular muscles particularly of the left medial rectus. On this treatment the proptosis and chemosis improved, but external ocular movements became more restricted: there was no abduction of the left eye, and on the right was restricted to $30^{\circ}$; elevation was restricted to $10^{\circ}$ on the left and $20^{\circ}$ in the right eye; down gaze was preserved.

The patient's diplopia continued to deteriorate and by early 1984 both eyes were totally immobile (Figure $2 a, 2 b)$. Initially, the right eye was in the neutral position but with further deterioration became fixed in adduction. Seven months after presentation, as prednisone was both ineffective and producing unacceptable side effects, the dose was reduced to $7.5 \mathrm{mg} / \mathrm{day}$ and
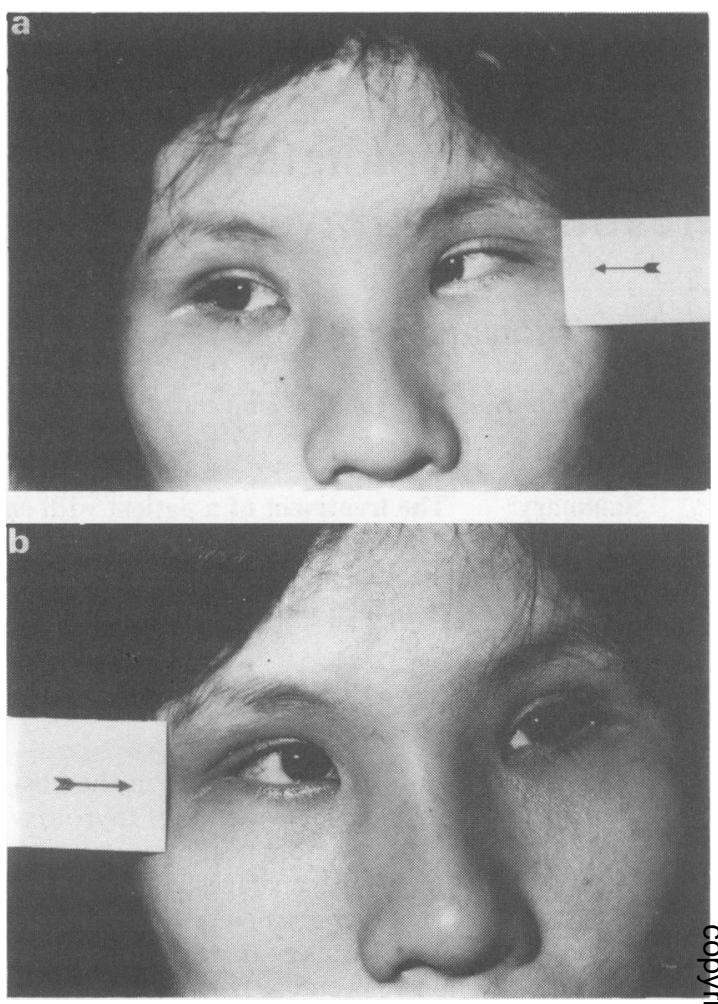

Figure 3 Eye movements after irradiation: (a) patient looking to her right; (b) patient looking to her left.

cyclophosphamide $(1 \mathrm{mg} / \mathrm{kg} / \mathrm{day})$ added with radiation of the orbits, sparing the lens $(2000 \mathrm{cGy}$ in ten fractions over 13 days).

The chemosis and proptosis subsided; the restriction of extra-ocular movements gradually improved. Five months after irradiation and on the same dose of cyclophosphamide and prednisone, the visual axes were now parallel; diplopia was now only present in the extremes of lateral gaze due to fibrotic shortening of both medial recti muscles (Figure 3a, 3b). A CT scan showed a marked reduction in size of the extraocular muscles (Figure 4). After 13 months, cyclophosphamide was stopped and prednisone reduced to $2.5 \mathrm{mg}$ a day. There has been no deterioration since.

\section{Discussion}

This patient exhibited all the features of autoimmune ophthalmopathy: chemosis, proptosis, limitation of eye movements and the pathognomonic CT changes of extraocular muscle swelling. ${ }^{8}$

The exact pathogenesis of the disease is unclear, but 


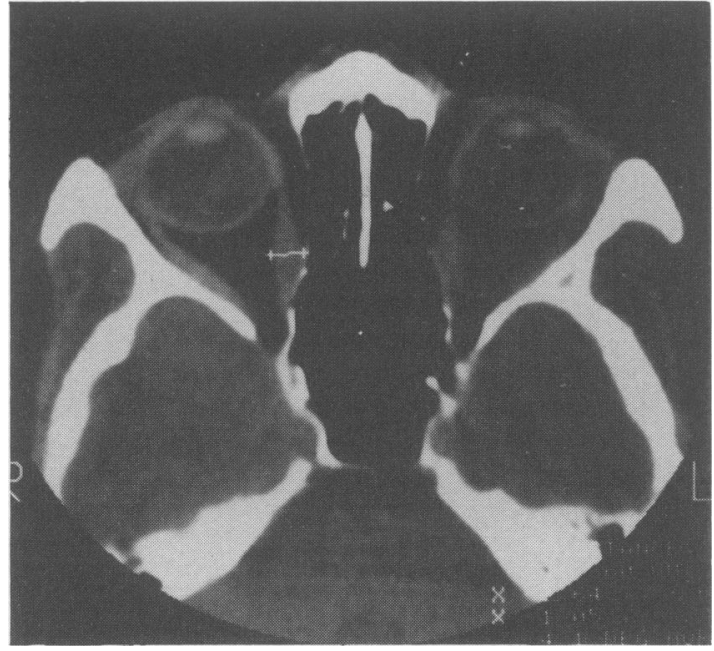

Figure 4 CT scan after orbital irradiation showing reduction in size of extraocular muscles.

both systemic as well as local orbital immunological processes have been implicated. The evidence includes the binding to extra-ocular muscles of immune complexes of thyroglobulin and its antibodies ${ }^{6}$, the finding of circulating antibodies directed against an

\section{References}

1. Bayliss, $\mathbf{R}$. Thyroid disease in the expression of autoimmune disease. The Harveian Oration, Royal College of Physicians, London, 1983.

2. Faryna, M., Nauman, J. \& Gardas, A. Measurement of auto-antibodies against human eye muscle plasma membranes in Graves' ophthalmopathy. Br Med J 1985, 290, 191-192.

3. Burde, R.M. The Orbit. In Lessels, L. \& Van Dalen, J.T.W. (eds). Neuro-ophthalmology, Vol. 2. Excerpta Medica, Amsterdam, 1982, pp 272-279.

4. Bartalena, L., Marcocci, C., Chiovato, L. et al. Orbital cobalt irradiation combined with systemic corticosteroids for Graves' ophthalmopathy: comparison with systemic corticosteroids alone. J Endocrinol Metab 1983, 56: 1139-1144.

5. Weetman, A.P., Ludgate, M., Mills, P.V. et al Cyclosporin improves Graves' ophthalmopathy. Lancet 1983, i, 486-489. antigen derived from oculomotor muscle ${ }^{9}$ and of T-cell mediated cytotoxicity to human ocular muscles. ${ }^{5}$

Treatment regimes have therefore been designed to counteract these processes and include the use of steroids, immunosuppressive agents, irradiation, plasmapheresis, and cyclosporin. ${ }^{3}$ However, the response to any of the above methods is variable. Steroids and irradiation in combination was reported to have a slightly better reponse than steroids alone. ${ }^{4}$ While cyclosporin was effective in some patients, ${ }^{5}$ in others, clinical deterioration developed during treatment. ${ }^{7}$

Recently, antibodies reacting with plasma membrane of extraocular muscles have been isolated from patients with Graves' ophthalmopathy, ${ }^{2}$ suggesting that there may be similarities to auto-immune myasthenia gravis. In the latter condition, cyclophosphamide and whole-body irradiation were curative in experimental myasthenia in mice. ${ }^{10}$ By analogy, the same regime was employed here, except that the irradiation was confined to the orbits and within 5 months, eye movements had completely recovered apart from limited abduction because of fibrosis of the medial rectus muscles.

Thus low dose cyclophosphamide and orbital irradiation can be effective in autoimmune ophthalmopathy; muscle involvement when present may be particularly responsive to this combined therapy. In the doses used, no side effects were observed.

6. Weetman, A.P., McGregor, A.M. \& Hall R. Ocular manifestations of Graves' disease: a review. J Roy Soc Med 1984, 77: 936-942.

7. Howlett, T.A., Lawton, N.F., Fells, P. \& Besser, G.M. Deterioration of severe Graves' ophthalmopathy during cyclosporin treatment. Lancet 1984, ii, 1101.

8. Werner, S.C., Coleman, D.J. \& Franzen, L.A. Ultrasonograph evidence of a consistent orbital involvement in Graves' disease. N Engl J Med 1974 290: 1447-1450.

9. Kodama, K., Sikorska, M., Bandy-Dafoe, P., Bayly, R. \& Wall, J.R. Demonstration of a circulating autoantibody against a soluble eye-muscle antigen in Graves' ophthalmopathy. Lancet 1982, ii, 1353-1356.

10. Pestronk, A., Drachman, D.B., Teoh, R.\& Adams, R.N. Combined short-term immunotherapy in experimental autoimmune myasthenia gravis. Ann Neurol 1983, 14: 235-241. 